\title{
Events of High Polymer Activity in Drag Reducing Flows
}

\author{
C. Tesauro • B. J. Boersma • M. A. Hulsen · \\ P. K. Ptasinski • F. T. M. Nieuwstadt
}

Received: 10 May 2006 / Accepted: 26 February 2007 /

Published online: 29 March 2007

(C) Springer Science + Business Media B.V. 2007

\begin{abstract}
The mechanism of drag reduction in turbulent flows due to polymers has been investigated with help of a direct numerical simulation. In particular, we consider the interaction between turbulent velocity fluctuations and polymers in terms of elastic energy that can be stored in the polymer. To this end all the terms of the elastic energy budget have been computed. The most interesting term is the production of elastic energy due to turbulent fluctuations, because it describes the interaction between polymers and turbulence. Although this term appears to be small in the average, it turns out that it can reach very large values instantaneously and intermittently, and the energy transfer from polymer to turbulence is located in very well defined areas inside the channel. This implies that locally there is a strong interaction between the polymer and the turbulent flow structure, and this strong interaction is mostly seen in areas of high velocity fluctuations.
\end{abstract}

Keywords Direct numerical simulation $\cdot$ FENE-P model $\cdot$ Energy balance

\section{Introduction}

It is well known that the addition of a small amount of polymers to a turbulent pipe or channel flow leads to a significant reduction of frictional drag. Since the discovery of this phenomenon by Toms [1], much work has been done (both by computations and experiments), but as yet no generally accepted explanation of this effect exists.

C. Tesauro $(\bowtie)$ B B. J. Boersma · P. K. Ptasinski · F. T. M. Nieuwstadt

J.M. Burgers Center for Fluid Dynamics, Delft University of Technology,

Leeghwaterstraat 21, 2628CA Delft, The Netherlands

e-mail: c.tesauro@wbmt.tudelft.nl

M. A. Hulsen

Materials Technology, Eindhoven University of Technology,

P.O. Box 513, 5600MB Eindhoven, The Netherlands 
In particular, two theories have been developed. In the first one, proposed by Lumley [2], the stretching of the polymer molecules by the flow increase the effective (extensional) viscosity. This leads to a thickening of the buffer layer and as a result to drag reduction. The second theory has been proposed by de Gennes [3], who has argued that drag reduction is caused by the elasticity of polymer molecules, by which energy at the small scales of turbulence is adsorbed and subsequently radiated away in form of shear waves.

Over the past years, many numerical simulations on drag reduction by polymers have been carried out with different kind of polymer models. (Den Toonder [4] and Orlandi [5], Massah et al. [6], Surehkumar [12], Dimitropoulos et al. [7], Ptasinski et al. [8]).

In this paper, the results of a direct numerical simulation with FENE-P model are presented for two cases of drag reduction and analysed for the effect of polymers on turbulence. In particular we focused on the interaction between polymers and turbulent structures in terms of the elastic energy that can be stored in the polymers.

\section{Governing Equations and Computational Procedures}

We consider a dilute solution of a Newtonian fluid, in which polymer molecules are dissolved. The solution is assumed to be dilute enough so that polymer molecules do not interact with each others, even when they are fully stretched. We consider an isothermal and incompressible flow, the dynamics of which can be described by the continuity equation and conservation of momentum in their dimensionless form. ${ }^{1}$

$$
\begin{aligned}
\nabla \cdot \mathbf{u} & =0 \\
\frac{\partial \mathbf{u}}{\partial t} & =-\mathbf{u} \cdot \nabla \mathbf{u}-\nabla p+\beta \frac{1}{R e_{*}} \nabla^{2} \mathbf{u}+\nabla \cdot \tau^{(\mathbf{p})}
\end{aligned}
$$

where $\mathbf{u}$ is the velocity vector, $p$ is the pressure, $\tau^{(\mathbf{p})}$ is the extra stress induced by the polymer molecules, $\beta=\eta_{s} / \eta_{0}$ is the ratio of the solvent viscosity $\eta_{s}$ to the total shear viscosity of the solution, $\eta_{0}$, at zero shear rate, and $R e_{*}=\rho u_{*} h / \eta_{0}$ is the Reynolds number.

The polymer stress tensor can be obtained by modeling the polymers using the FENE-P (finite elastic non linear extensible) model with Peterlin approximation: a polymer chain is represented as two spherical beads connected by a massless spring. The dumbbell is specified by a connector vector $\mathbf{Q}$, which describes the orientation and the internal configuration of the polymer as illustrated in Fig. 1. This model is characterized by a maximum length $Q_{0}$ and a relaxation time $\lambda$. With help of a force balance on the center of mass of the dumbbell we can derive an evolution equation for the conformation tensor in dimensionless form $\mathbf{c}=\langle\mathbf{Q Q}\rangle /(k T / H)$ (where $\sqrt{k T / H}$, with $H$ the spring constant, $k$ the Boltzmann constant and $T$ the

\footnotetext{
${ }^{1}$ All equations are made dimensionless using the friction velocity $u_{*}$ that can be expressed in terms of density and wall shear stress: $u_{*}=\sqrt{\tau_{w} / \rho}$, and the channel height $h$.

悬 Springer
} 
Fig. 1 Schematic

representation of a dumbbell

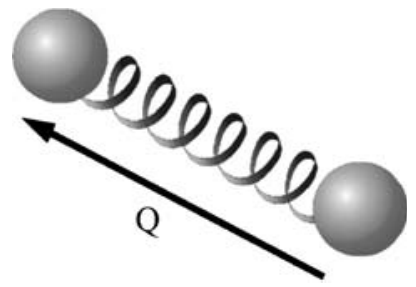

absolute temperature, is a scale length and $\langle\cdots\rangle$ denote an average over all possible conformations),

$$
\frac{\partial \mathbf{c}}{\partial t}=-\mathbf{u} \cdot \nabla \mathbf{c}+\nabla \mathbf{u} \cdot \mathbf{c}+\mathbf{c} \cdot(\nabla \mathbf{u})^{\mathrm{T}}+\frac{1}{\tilde{\lambda}}\left(\mathbf{I}-\frac{1}{1-\frac{\operatorname{tr} \mathbf{c}}{b}} \mathbf{c}\right)
$$

where $b=H Q_{0} / k T$ is a length parameter and $\tilde{\lambda}=\lambda u_{*} / h$ is the dimensionless relaxation time of the dumbbell, which can be expressed as a dimensionless Weissenberg number which gives the ratio between the characteristic time-scale of the polymer and a characteristic time-scale of the flow $W e_{*}=R e_{*} \tilde{\lambda}$.

Details about this model can be found in Bird et al. $[9,10]$.

The extra stress of the polymer can be computed as function of the polymer conformation tensor:

$$
\tau^{(p)}=n k T\left(-\mathbf{I}+\frac{1}{1-\frac{\operatorname{tr} \mathbf{c}}{b}} \mathbf{c}\right)
$$

The system of (1), (2) and (3) is discretized with help of a pseudo-spectral method in the streamwise and spanwise directions (in these directions periodic boundary conditions are applied) and a staggered finite difference method in the wall-normal direction. On the channel walls no-slip boundary conditions are applied. The simulation is carried out in a so-called minimal flow unit (MFU, see Jimenez and Moin [11]) with dimensions $1.5 h$ in the streamwise, $h$ in the spanwise and $h$ in the wall normal direction (Fig. 2).

The momentum equations are integrated in time using a second-order AdamsBashforth method for the advective, diffusive and polymer terms with the standard pressure correction method to enforce continuity. The time step is determined using the Courant criterion.

For the integration of the discretized the equation for the polymer conformation tensor, which is solved simultaneously with the continuity and the momentum equations, we use a second-order Adams-Bashforth scheme for the advection and the deformation due to the flow and a second-order Adams-Moulton scheme for

Fig. 2 Schematic representation of the geometry used in the DNS

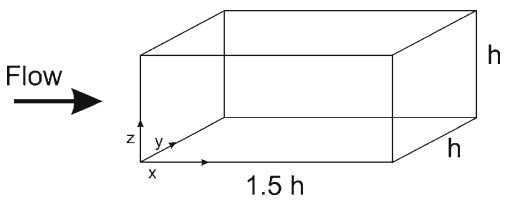


Table 1 Numerical parameters of the FENE-P model used in the direct numerical simulations

\begin{tabular}{lllcc}
\hline Run & $\beta$ & $W e_{*}$ & $b$ & $\% D R$ \\
\hline $\mathrm{N}$ & 1 & - & - & - \\
$\mathrm{A}$ & 0.6 & 54 & 100 & 26 \\
$\mathrm{~B}$ & 0.6 & 54 & 1,000 & 61
\end{tabular}

"N" indicates the DNS of a Newtonian flow.

the FENE-P force in order to prevent the dumbbells from exceeding their maximum length, i.e. $\operatorname{tr} \mathbf{c}<b$. To avoid numerical instabilities a small artificial diffusive term $a /\left(u_{*} h\right) \nabla^{2} \mathbf{c}$ is added to the equation for the conformation tensor (3), in our case we have taken $a /\left(u_{*} h\right)=1.2 \times 10^{-2}$ (see Sureshkumar et al. [12]). The effect of this diffusive term should decrease when the number of grid points and the time step decrease and it should not have big influence on the macroscopic flow parameters like the velocity or stress profiles. The value chosen in this paper has been found to not affect in a significant way the results of those parameters: an analysis carried on by Ptasinski et al. [8] showed that for two different DNS of the same flow field using two different values of $a /\left(u_{*} h\right)=\mathcal{O}\left(10^{-2}\right)$ the velocity and stress profiles did not show significant differences.

Details about the computational procedures used in the DNS can be found in Ptasinski et al. [8].

The computations are carried out on a grid with $48 \times 32 \times 100$ grid points in the $x$ - (streamwise), $y$ - (spanwise) and $z$ - (wall normal) directions respectively and with a dimensionless time step of $\Delta t=2 \cdot 10^{-4}$ for the Newtonian and $\Delta t=1 \cdot 10^{-4}$ for the viscoelastic case. The results for Newtonian flow agree very well with the results of Kim et al. [13], which are considered as the standard reference for direct numerical simulations of channel flow at low Reynolds numbers.

We have carried two simulations both with $R e_{*}=360$ but with a varying the length parameter $b$ for the polymer. An overview of the numerical parameters for which computations have been carried out, is given in Table 1 , where the amount of drag reduction (in percentage) is expressed as the increase of flow rate due to the addition of the polymers with respect to Newtonian flow (see Gyr and Bewersdorff [14]).

\section{Elastic Energy of the Polymers}

We focused in this paper on the potential energy stored in the elastic polymers. In an elastic spring model, where the connector force given by $\mathbf{F}=\mathbf{F}(\mathbf{Q})$ with $\mathbf{Q}$ the extension of the spring, the elastic energy $\phi$ is equal to:

$$
\phi=\int \mathbf{F}(\mathbf{Q}) \mathbf{d} \mathbf{Q}
$$

The equation for the elastic energy balance is given by:

$$
\frac{D \phi}{D t}=\frac{\partial \phi}{\partial t}+u_{j} \frac{\partial \phi}{\partial x_{j}}=\tau_{i j}^{(p)} \frac{\partial u_{i}}{\partial x_{j}}-\frac{1}{2 \tilde{\lambda}} \frac{1}{1-\frac{\operatorname{tr} \mathbf{c}}{b}} \tau_{i i}^{(p)}
$$


Applying the Reynolds decomposition (i.e. $\phi=\Phi+\phi^{\prime}, \tau_{i j}^{(p)}=\mathcal{T}_{i j}^{(p)}+\tau_{i j}^{\prime(p)}$ and $\left.u_{i}=U_{i}+u_{i}^{\prime}\right)$ to this equation leads to

$$
\begin{aligned}
\frac{D \bar{\Phi}}{D t} & =\frac{\partial \bar{\Phi}}{\partial t}+U_{j} \frac{\partial \bar{\Phi}}{\partial x_{j}}= \\
& =-\frac{\partial}{\partial x_{j}} \overline{\left(u_{j}^{\prime} \phi^{\prime}\right)}+\mathcal{T}_{i j}^{(p)} \frac{\partial U_{i}}{\partial x_{j}}+\overline{\tau_{i j}^{\prime(p)} \frac{\partial u^{\prime}}{\partial x_{j}}}-\frac{1}{2 \tilde{\lambda}} \overline{\frac{1}{1-\frac{\operatorname{tr} \mathbf{c}}{b}} \tau_{i i}^{(p)}}
\end{aligned}
$$

The first term on right hand side of (7) denotes the transport of elastic energy by velocity fluctuation, the second and third are respectively the production of elastic energy due to mean and turbulent flow, the last is a dissipative term, which can be interpreted as the transfer of energy into heat by relaxation of the polymers from an extended state to their equilibrium state. In particular the term $\overline{\tau^{\prime(p)} \partial u_{i}^{\prime} / \partial x_{j}}$ is of interest here because it describes the aforementioned interaction between the polymers and the turbulence, i.e. the same term (with an opposite sign) is also present in the equation for the turbulent kinetic energy. When this term is positive, the energy is removed from the flow and stored in the polymers and vice versa for a negative sign. In the latter case polymers produce turbulent kinetic energy.

\section{Results and Discussion}

In Fig. 3 we show the results for the mean velocity profiles for all the polymer cases and for the Newtonian case. For a small value of the extensibility parameter ( $b=100$, run A) we found only a small amount of drag reduction. Increasing this parameter (to $b=1,000$ and $W e_{*}=54$, run $\mathrm{B}$ ) the drag reduction increases to

Fig. 3 Profiles of mean streamwise velocity as function of the distance from the wall

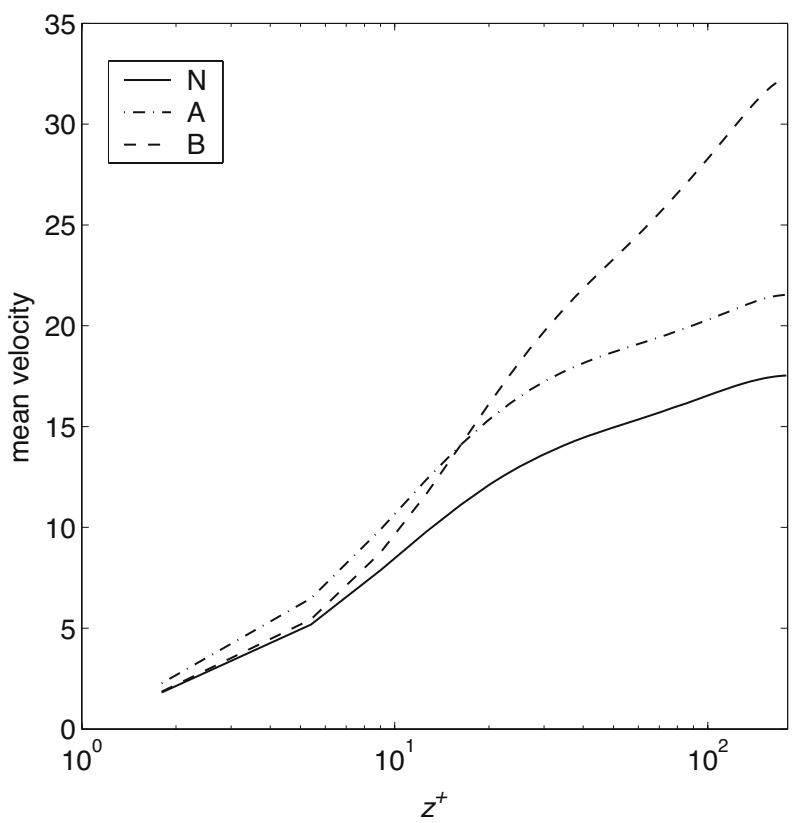

Springer 

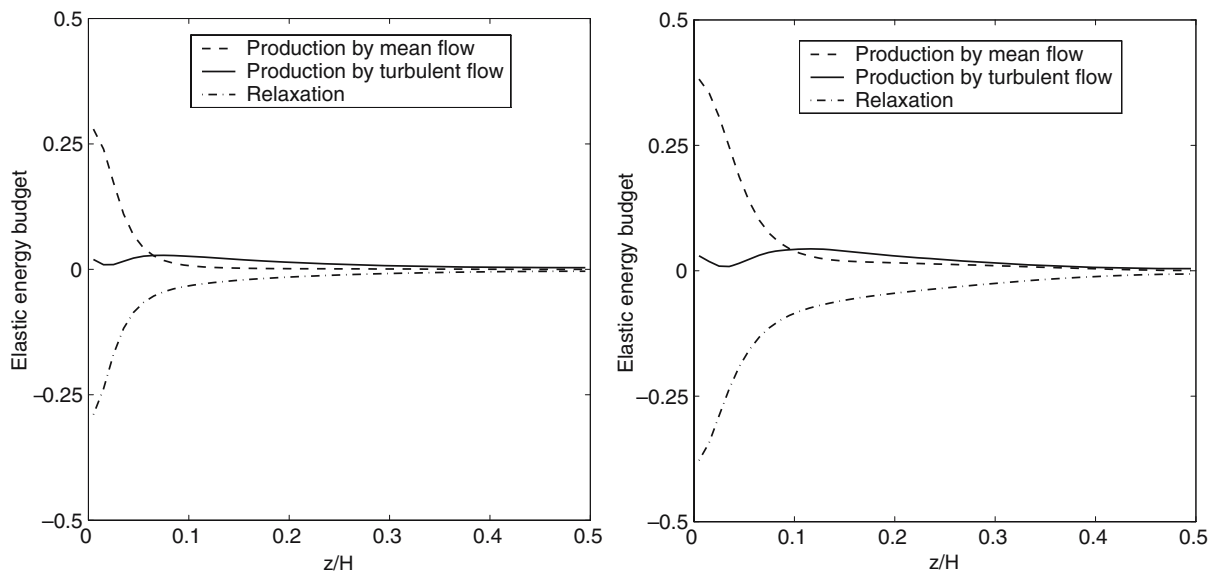

Fig. 4 Polymer elastic energy budget as function of the distance from the wall for case A (left) and $\mathrm{B}$ (right)

a value close to the maximum drag reduction asymptote. For case A $(b=100)$ the buffer layer extends to a larger distance from the wall in comparison with the Newtonian case and the profile in the logarithmic layer is shifted upwards parallel with respect to the profile of the Newtonian simulation. For case $\mathrm{B}(b=1,000)$ the buffer layer extends almost across the whole channel, which is characteristic for maximum drag reduction.

In Fig. 4 we have plotted the various contributions to the elastic energy budget for the two polymer cases. The transport term has not been included in the figure because it was found to be negligible. Close to the wall the elastic energy balance reduces to a difference between two large terms, i.e. mean flow production and dissipative relaxation, while further away from the wall, i.e. in the buffer layer and beyond,
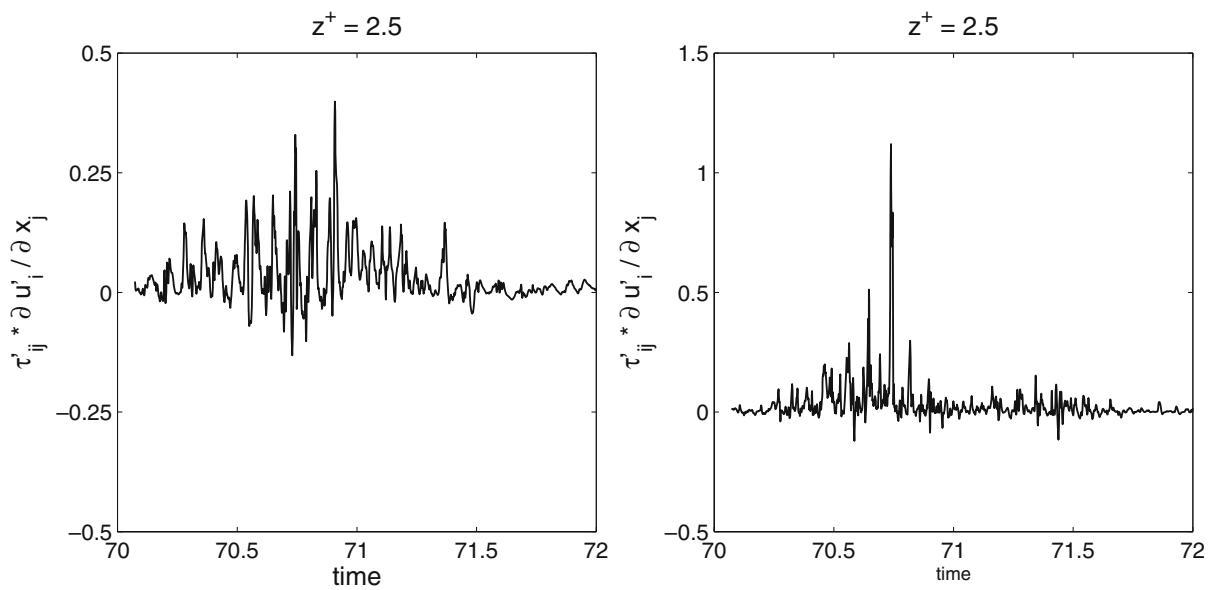

Fig. 5 Time history of the production of elastic energy due to turbulent flow, case A Springer 

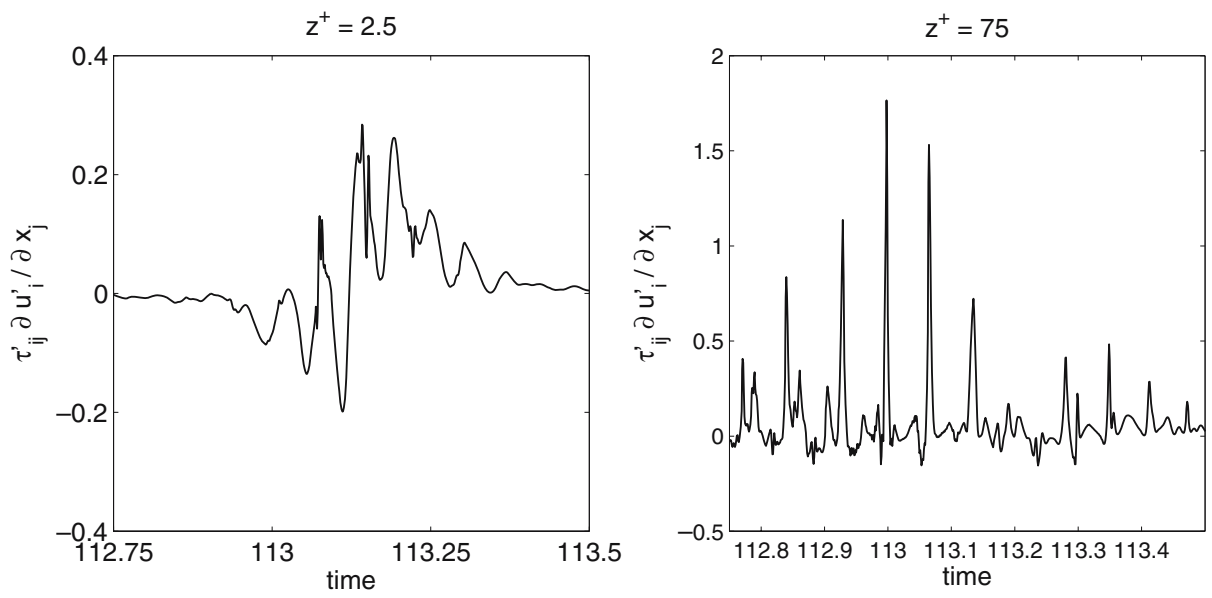

Fig. 6 Time history of the production of elastic energy due to turbulent flow, case B

the production by the fluctuating velocity field becomes the dominant term although its magnitude is much smaller than the value of mean flow production near the wall.

When we look to the instantaneous value of the production of elastic energy due to turbulent fluctuation, the situation becomes quite different. In Figs. 5 and 6 we show the time-series of $\overline{\tau_{i j}^{\prime(p)} \partial u^{\prime} / \partial x_{j}}$ at single position in the channel and at two different distances from the lower wall. We see that interaction between turbulence and polymer can instantaneously and intermittently reach very large values, which implies a strong local interaction between turbulence and polymers. The low mean value of the turbulent production term is then due to a cancellation of positive and negative peaks. The biggest excursions have been found in the buffer layer, and because for high drag reduction regime the buffer layer extend almost across the whole channel, big excursions are found for case B also at higher distance from the wall. The oscillating behavior of the timeseries can be explained by the periodic boundary condition of the flow in the streamwise direction: the period of the oscillation is in

Fig. 7 Comparison between the production of elastic energy due to turbulent flow for case B for two different channels

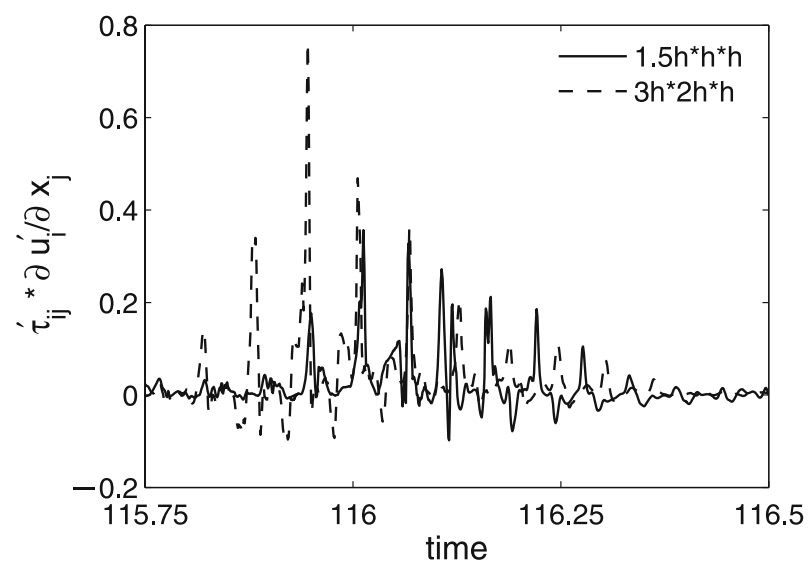


Fig. 8 Isosurfaces of $\tau_{i j}^{\prime(p)} \partial u_{i}^{\prime} / \partial x_{j}$ for case A (up) and $\mathrm{B}$ (down). The gray areas is where energy is transferred form the turbulence to the polymers $\left(\tau_{i j}^{\prime(p)} \partial u_{i}^{\prime} / \partial x_{j}>0\right)$, while the black areas is where energy is transferred from the polymers to the turbulence $\left(\tau_{i j}^{\prime(p)} \partial u_{i}^{\prime} / \partial x_{j}<0\right)$

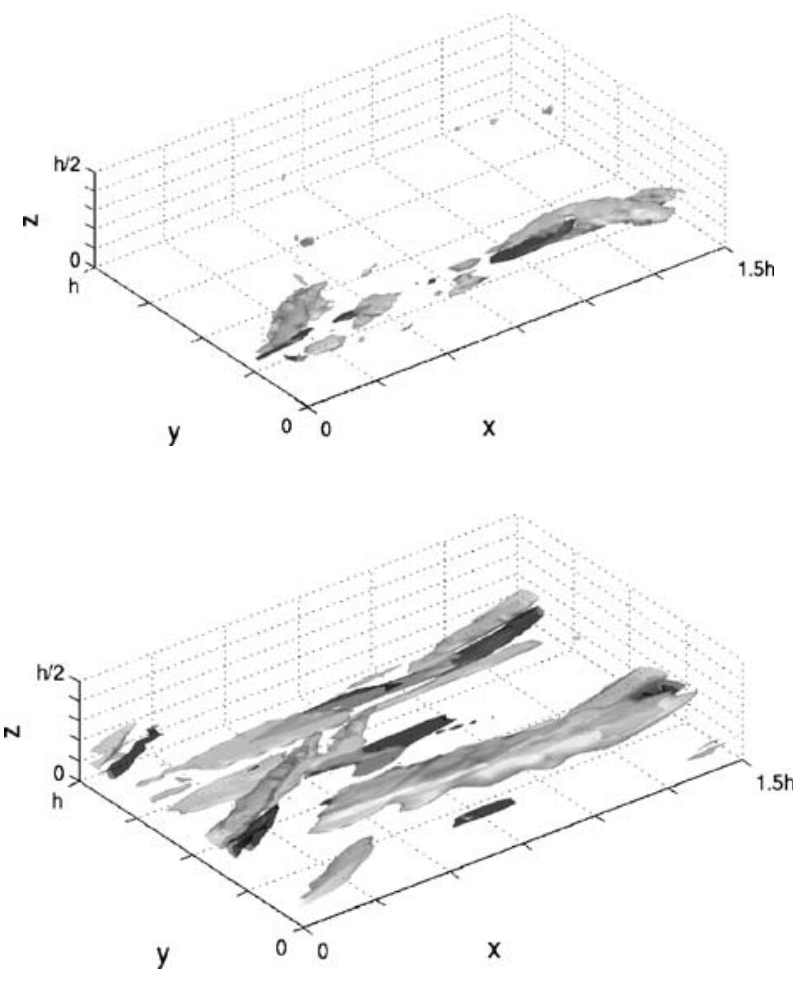

fact related to the bulk velocity of the flow. An extra DNS has been carried out with a a bigger domain size $(3 h \times 2 h \times h)$ using 96 grid points in the streamwise, 64 grid points in the spanwise and 100 grid points in the wall normal direction) for case $B$. The comparison of the timeseries for the two DNS is shown in Fig. 7: in both cases, the same oscillating behaviour can be seen, and no big difference have been found in the values reached by the production of elastic energy due to velocity fluctuation.

In Fig. 8 we show the isosurfaces of the turbulence-polymer interaction term for both DNS. An area of positive value for the interaction term occurs side by side with an area of negative values. In agreement with Figs. 5 and 6 we find that the region of strong interaction becomes larger for the case with the largest extensibility parameter $b$. This implies that the exchange of energy from turbulence to polymers and vice versa is located on very well defined areas of the channels, where streamwise velocity fluctuations are normally high. Similar results have also been found by Dubief et al. [15], in which work it has been found that the energy transfer is also located in very well defined areas close to the near wall regions. In this paper however we showed that at high drag reduction the energy transfer can be a really important term also farther away from the wall, because in the high drag reduction regime velocity fluctuations are high also in the buffer layer.

An intermittent added dissipation have been found by Massah and Hanratty [16], who computed the added stresses and the added dissipation associated with the movement of FENE-P chains in a velocity field obtained from a DNS of turbulent flow of Newtonian fluid in a channel, the intermittent added dissipation is associated 
with an increase of the size of the wall vortices in producing Reynolds stresses. In this paper we found the intermittent added dissipation also in a more complex flow and using a simulation technique that takes into account also the effects of the polymers on the flow, and we showed that the energy exchange is located in very well defined areas of high velocity fluctuations.

\section{Conclusions}

We have carried out a DNS of dilute polymer solution in turbulent flow. Polymers are modeled as elastic dumbbells with FENE-P approximation, and the equation of motion and the evolution equation for polymers are solved simultaneously with a twoway coupling, i.e. polymers are stretched by the velocity field and the polymer stress is then returned in the flow equations. The flow is isothermal and incompressible. Two DNS have been carried out at two different values of the extensibility parameter $b$, which is proportional to the square of the maximum length of the polymers, in order to reach two different rate of drag reduction.

The profiles of the mean streamwise velocity are found to shift upwards with respect to the Newtonian profile for both DNS, at high drag reduction a change in the slope of the profile has also been found. The peak of the r.m.s of the streamwise velocity fluctuations increased with increasing the extensibility parameter $b$ with respect to the value for Newtonian flow. At high drag reduction level, the location of the peak is also shifted away from the wall, as a result of a thickening of the buffer layer.

The results for the elastic energy budget show that the interactions between polymers and turbulence appears to be small in an average balance, but it can instantaneously and intermittently reach very large values, which implies a strong local interaction between turbulence and polymers. Because of the thickening of the buffer layer, the highest peaks are found farther away from the wall in the high drag reduction case, in areas where streamwise velocity fluctuations are high. The amount of interaction between polymers and turbulence has been found to be dependent on the extensibility parameter $b$, which suggests that extensibility is an important parameter for polymer drag reduction.

\section{References}

1. Toms, B.A.: Some observations of the flow of linear polymer solutions though straight tubes at large Reynolds numbers. In: Proceedings of the 1st International Congress on Rheology, pp. 135-141. North Holland, Amsterdam (1949)

2. Lumley, J.L.: Drag reduction by polymer additives. Annu. Rev. Fluid Mech. 1, 367-384 (1969)

3. De Gennes, P.G.: Introduction to Polymer Dynamics. Cambridge University Press, Camridge (1990)

4. Den Toonder, J.M.F., Hulsen, M.A., Kuiken, G.D.C., Nieuwstadt, F.T.M.: Drag reduction by polymer additives in a turbulent pipe flow: numerical and laboratory experiments. J. Fluid Mech. 337, 193-231 (1997)

5. Orlandi, P.: A tentative approach to direct numerical simulation of drag reduction by polymers. J. Non-Newton. Fluid Mech. 60, 277-301 (1995)

6. Massah H., Kontomaris, K., Schowalter, W.R., Hanratty, T.J.: The configurations of a FENE bead-spring chain in transient rheological flows and turbulent flow. Phys. Fluids 5, 881-889 (1993)

7. Dimitropoulos, D.D., Sureshkumar, R., Beris, A.N.: Direct numerical simulation of viscoelastic turbulent flow exhibiting drag reduction: effect of the variation of rheological properties. J. NonNewton. Fluid Mech. 79, 433-468 (1998) 
8. Ptasinski, P.K., Boersma, B.J., Nieuwstadt, F.T.M., Hulsen, M.A., van den Brule, B.H.A.A., Hunt, J.C.R.: Turbulent channel flow near maximum drag reduction: simulations, experiments and mechanisms. J. Fluid Mech. 490, 251-291 (2003)

9. Bird, R.B., Curtiss, C.F., Armstrong, R.C., Hassager, O.: Dynamics of Polymer Liquids, vol. 2, 2nd edn. Wiley, New York (1987)

10. Bird, R.B., Dotson, P.J., Johnson, N.L.: Polymer solution rheology based on a finitely extensible bead-spring chain model. J. Polym. Sci., B, Polym. Lett. 7, 213-235 (1980)

11. Jimenez, J., Moin, P.: The minimal flow unit in near-wall turbulence. J. Fluid Mech. 225, 213-240 (1991)

12. Sureshkumar, R., Beris, A.N., Handler, R.A.: Direct numerical simulation of turbulent channel flow of polymer solution. Phys. Fluids 9, 743-755 (1997)

13. Kim, J., Moin, P., Moser, R.: Turbulence statistics in fully developed channel flow at low Reynolds number. J. Fluid Mech. 177, 133-166 (1987)

14. Gyr, A., Bewersdorff, H.-W.: Drag Reduction of Turbulent Flows by Additives. Kluwer, Dordrecht (1995)

15. Dubief, Y., White, C.M., Terrapon, V., Shaqfeh, E.S.G., Moin, P., Lele, S.K.: On the coherent drag-reducing and turbulence enhancing behaviour of polymer in wall flows. J. Fluid Mech. 514, 271-280 (2004)

16. Massah, H., Hanratty, T.: Added stresses because of the precence of FENE-P bead-spring chains in a random velocity field. J. Fluid Mech. 337, 67-101 (1997) 